\title{
The Failure of the Cross Correlation Measurement Technique
}

\author{
Ken McGill, Kathryn Harke, Kris Schock \\ Department of Chemistry, Physics, and Astronomy, Georgia College and State University, Milledgeville, GA, \\ USA \\ Email: ken.mcgill@gcsu.edu
}

Received 17 February 2015; accepted 7 March 2015; published 10 March 2015

Copyright (C) 2015 by authors and Scientific Research Publishing Inc.

This work is licensed under the Creative Commons Attribution International License (CC BY). http://creativecommons.org/licenses/by/4.0/

c) (i) Open Access

\begin{abstract}
The experiment involves creating a sound wave that propagates down a pipe with 8 transducers attached at equally spaced intervals of $0.01016 \mathrm{~m}$. The numerical method-the Cross Correlation Method, used to solve for the phase component, creates a high correlation value, but the speed of sound varies immensely. The method involves a Fast Fourier Transform (FFT) of the collected data, which is used to find the phase of the sound wave, and the slope of the position versus time graph, which is used to calculate the speed of sound. This high correlation value shows that the data are correct, but the numerical method for analyzing the data is incorrect.
\end{abstract}

\section{Keywords}

\section{Acoustic Array, Cross Correlation, Flow Measurement}

\section{Introduction}

Since the establishment of the first oil transporting pipeline system in 1879 , half a million miles of pipe line networks have been built across the United States [1]. With the rising nationwide demand for various commodities such as water, petroleum, and natural gas, the expansion of the pipeline system was a necessity as a means of transportation for millions of dollars of valuable merchandise. With the growing number of pipelines nationwide and the increasing volume of liquids transported daily via pipeline, a newfound need has arisen for technology that accurately calculates the rate of flow of these liquids. In order to control the amount of a substance sent through a pipe, the measure of its flow velocity must be determined to a high level of accuracy. Various techniques and technologies have been created in an attempt to provide an accurate measurement for flow in a closed conduit; however, they offer results with varying degrees of inaccuracies. The Cross Correlation Method has become a popular method in determining rate of flow through a pipeline [2]. A proposed acoustic method 
employs a set of transducers along the measured pipe which detect time delay for an acoustic wave propagating through the pipe.

\section{Theory}

\section{The Wave Equation}

A solution to the wave equation for a sound wave propagating through a pipe is:

$$
\Psi=A \sin \left(k(x+\pi \sin )=\pi A \sin \left(\frac{2 \pi}{\lambda}(x+\lambda f t)\right)=A \sin \left(2\left(\frac{x}{\lambda}+f t\right)\right)=A \quad(\theta+f t)\right.
$$

where $A$ is the normalization constant, $k$ is $(2 \pi) / \lambda, f$ is the frequency of the wave, $t$ is the time, $x$ is the position, $\lambda$ is the wavelength of the wave, and $\theta$ is the phase angle of the wave propagating through space [3]. By comparing the arguments of the functions sin ( ) in equality (1), one can see that the phase plotted versus the position will include the speed of sound [3].

$$
\begin{gathered}
\theta=\underset{\lambda}{2 \pi \underset{\lambda}{2} \pi}=\frac{f x}{c} \\
m=\frac{2 \pi f}{c}
\end{gathered}
$$

where $m$ is the slope of the line. Thus, the speed of sound, $c$, can be found as follows:

$$
c=\frac{2 \pi f}{m}
$$

\section{Procedure}

In an experiment to test this proposed method, 450 distinct frequencies of sound waves from 500 to $5000 \mathrm{~Hz}$ were sent down a $7.50 \mathrm{~m}$ long metal pipe with a $0.1524 \mathrm{~m}$ diameter Figure 1 . The range of 500 to $5000 \mathrm{~Hz}$ was chosen since any frequency below $500 \mathrm{~Hz}$ has interference due to a large amount of ambient noise, which could interfere with the results and $5000 \mathrm{~Hz}$ was the maximum frequency possible with the sample rate of 10,000

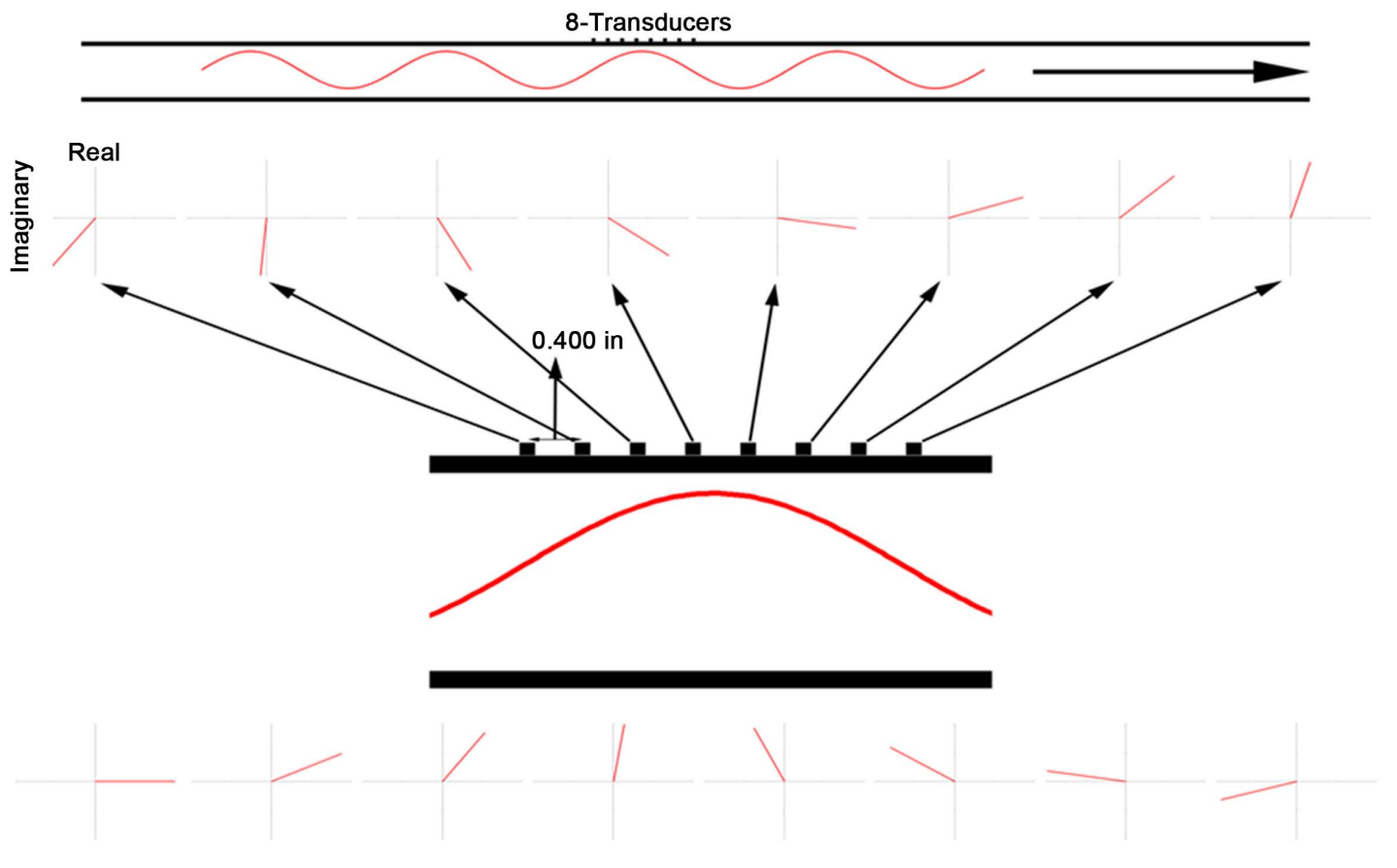

Figure 1. The acoustic array with 8 microphones. 
samples/s due to the Nyquist limit. The sound wave was measured using 8 transducers $0.01016 \mathrm{~m}$ apart. Since the minimum frequency used was $500 \mathrm{~Hz}$, the distance between the microphones needed to be larger than the maximum wavelength; thus, $0.01016 \mathrm{~m}$ (equivalent to $0.4 \mathrm{in}$ ) was chosen due to the precision of the machinery available. The 450 data points taken from the instrument were then converted using an analog-digital converter (ADC) as seen in the block diagram in Figure 2. In order to determine the real and imaginary components of the phase, a Fourier Analysis was conducted on the data obtained from experimentation. In order to obtain a value for the speed of sound, the phase was plotted versus the position and the slope was calculated (Figure 2). The speed of sound along with the correlation coefficients for all frequencies from 500 to $5000 \mathrm{~Hz}$ was then plotted.

\section{Data and Results}

While the results obtained from experimentation yield a high correlation coefficient between the phases of the sound wave and the position along the pipe, the value obtained for the speed of sound is incorrect even at correlation coefficients of 0.999 . The proposed cross-correlation method for determining the speed of a sound wave propagating through a pipe has been proven invalid for its intended purposes.

\section{Conclusions}

Based on the data obtained from experimentation, the Cross-Correlation Phase Measurement Technique has proven itself an inaccurate method in determining the speed of a sound wave propagating through a pipe at various frequencies. Even with a consistently high correlation coefficient between the phases of the sound wave and the position along the pipe, across the range of tested frequencies, the calculated speed of sound was inaccurate at a majority of the points (Figure 3). As seen in Figure 3, the range of frequencies: $1797 \mathrm{~Hz}-2197 \mathrm{~Hz}$, has a correlation coefficient close to 1 ; however, the speed of sound measured in $\mathrm{m} / \mathrm{s}$ has a range of values from 207.1 $\mathrm{m} / \mathrm{s}$ to $634.6 \mathrm{~m} / \mathrm{s}$. The speed of sound in air at room temperature should be $343 \mathrm{~m} / \mathrm{s}$ [4]. The correlation coefficient and the incorrect data suggest a flaw in the Cross-Correlation Measurement Technique. The flawed methodology behind this Cross-Correlation Phase Measurement Technique has neglected the presence of the reflected wave moving through the pipe in its calculations, thus resulting in inaccurate results. The inaccurate results are due to the superposition of the waves.

This technique does not take the superposition theory into account, but assumes that the input is coming from only one direction. Superposition of a sound wave describes a state of interference between multiple sound waves. This interference can be either constructive, where the waves are additive, or destructive, where the waves are reductive. This effect can be seen in the reflection of a sound wave in the pipe when two waves combine to form one superimposed sound wave. To illustrate the magnitude of effect superposition has on the results, consider the wave shown below:

$$
\left.\Psi=\cos (20) .0\left(\sin \frac{x}{c+v}\right)\right)+\quad\left(f\left(t-\frac{x}{c+v}\right)\right)
$$

where $f$ is the frequency; $c$ is the speed of sound; and $v$ is the flow velocity, which adds to $c$ when the flow velocity is in the same direction as the propagating wave. The equation assumes the presence of the same wave from another source, $90^{\circ}$ out of phase with $1 \%$ amplitude of the above wave. The resulting wave would have

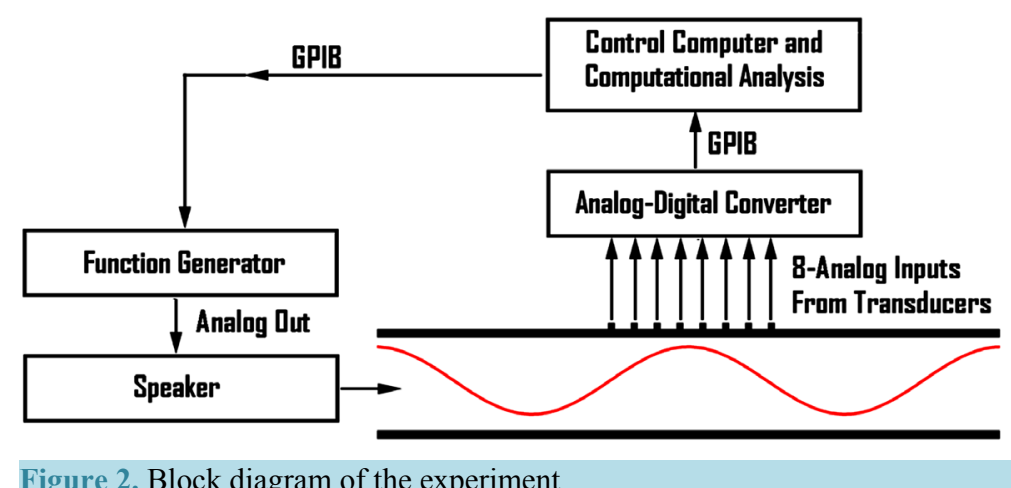

Figure 2. Block diagram of the experiment. 


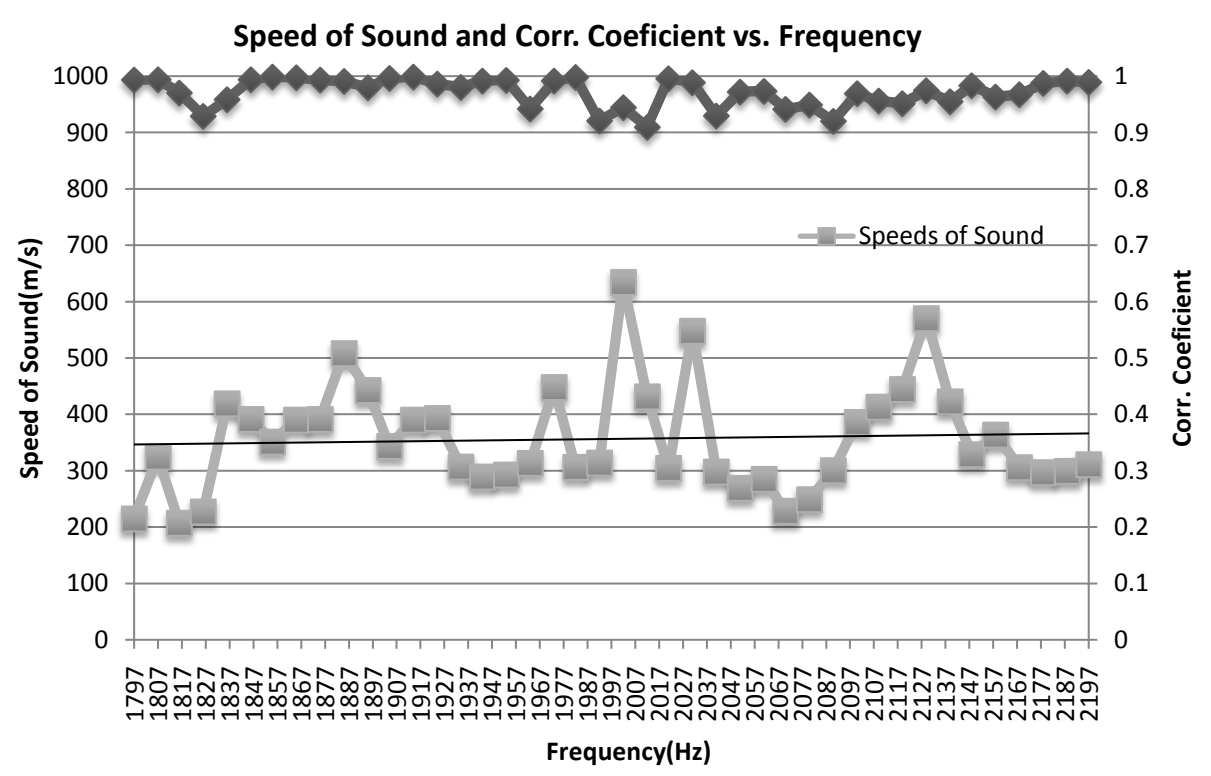

Figure 3. Speed of sound and correlation coefficient versus frequency.

a phase difference of $0.57=\tan ^{-1}(0.01)$ or $0.16 \%$ full scale. While $0.16 \%$ may seem small, this slight discrepancy translates into a notable error of $0.56 \mathrm{~m} / \mathrm{s}$ for a speed of sound of $350 \mathrm{~m} / \mathrm{s}$, and given a full range of flow velocity from $0 \mathrm{~m} / \mathrm{s}$ to $10 \mathrm{~m} / \mathrm{s}$, it translates to $5.6 \%$ error in flow velocity.

To give an idea of magnitude by converting the interfering wave amplitude to the input wave amplitude, a 40 $\mathrm{dB}$ difference is revealed in sound. This is equivalent to the sound level in a library. It is very likely that noise of this level, or more, would be present from the ambient noise in the pipe or from reflected waves.

$$
\frac{I}{I_{o}}=\left(\frac{\Delta A}{A}\right)^{2}=0.0001 \text {, or } \beta=10 \log \left(\frac{I}{I_{o}}\right)=-40 \mathrm{~dB}
$$

To employ acoustics to measure this phase phenomenon, it will be necessary to detect and separate out any miscellaneous background sound waves in order to more accurately analyze the sound wave present in the pipe. If the sound can be separated, it may be possible to use the ambient noise for the phase measure, and thereby remove the need for a sound source.

\section{Acknowledgements}

We would like to thank Georgia College and State University for providing the materials and space needed for this research.

\section{References}

[1] Kennedy, J.L. (1993) Oil and Gas Pipeline Fundamentals. 2nd Edition, PennWell Books, Oklahoma, 3-5.

[2] Beck, M.S. and Plaskowski, A. (1987) Cross Correlation Flowmeters, Their Design and Application. Taylor \& Francis, UK.

[3] Taylor, J.R. (2005) Classical Mechanics. University Science Books, Sausalito, 688.

[4] Kirkpatrick, L. and Francis, G. (2010) Physics: A Conceptual World View. 7th Edition, Brooks/Cole, Cengage Learning, California, 331. 